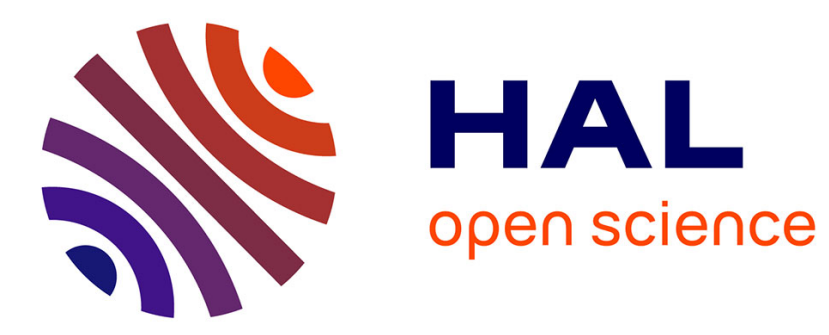

\title{
L'espace domestique des familles populaires: l'enfant vu comme acteur du groupe familial
}

Bernadette Tillard

\section{To cite this version:}

Bernadette Tillard. L'espace domestique des familles populaires: l'enfant vu comme acteur du groupe familial. Le Télémaque. Philosophie, Education, Société, 2014, 2, pp.135 - 151. 10.3917/tele.046.0135 . halshs-01228866

\section{HAL Id: halshs-01228866 \\ https://shs.hal.science/halshs-01228866}

Submitted on 24 Nov 2015

HAL is a multi-disciplinary open access archive for the deposit and dissemination of scientific research documents, whether they are published or not. The documents may come from teaching and research institutions in France or abroad, or from public or private research centers.
L'archive ouverte pluridisciplinaire HAL, est destinée au dépôt et à la diffusion de documents scientifiques de niveau recherche, publiés ou non, émanant des établissements d'enseignement et de recherche français ou étrangers, des laboratoires publics ou privés. 


\title{
L'espace domestique des familles populaires : l'enfant vu comme acteur du groupe familial.
}

\author{
Bernadette TILLARD, CLERSE - UMR 8019
}

\section{Résumé}

Comme le soulignent Esther Goody, parmi les responsabilités qui incombent aux parents, ceux-ci remplissent quotidiennement auprès de leurs enfants des tâches d'alimentation, d'apprentissage et d'entretien de la vie qui participent à leur éducation. Les logements précaires et les faibles revenus des familles de milieux populaires ont une influence sur la manière d'organiser la vie familiale. L'enfant y est sollicité pour participer à la vie domestique, tant dans l'entretien du logement que dans la garde des plus jeunes. Nous relevons dans nos différents terrains auprès de familles populaires du Nord de la France quelques particularités de l'éducation des enfants et leurs répercussions sur le statut de l'enfant dans sa famille.

\section{Introduction}

Depuis 1999, le terme de «parentalité » est largement utilisé dans le domaine du travail social. Cet anglicisme a fait l'objet de travaux anthropologiques auxquels nous nous référons et qui avaient pour ambition de définir un cadre général permettant de comparer les attributions des adultes, considérés comme «parents » dans l'engendrement et/ou l'éducation de l'enfant jusqu'à son entrée dans l'âge adulte. Comme Émile Durkheim, Esther Goody ${ }^{1}$ situe la parentalité dans la question plus globale de la reproduction sociale. C'est un aspect qui est peu relevé quand ses travaux sont cités en dehors de la sphère socio-anthropologique. Or, c'est pourtant bien là, une des spécificités du regard anthropologique qu'elle porte sur la parentalité dans son chapitre $A$ Framework for the analysis of parent roles du livre Parenthood and social reproduction. Fostering and occupational roles in West Africa $(1982)^{2}:$ « Les données anthropologiques sur la parentalité ont eu tendance soit à se concentrer sur les institutions formelles [...] soit à se préoccuper des relations émotionnelles précoces, de la prise en charge des soins et de

\footnotetext{
${ }^{1}$ Goody E. (1982). Parenthood and social reproduction. Fostering and occupational roles in West Africa. Cambridge, Cambridge University Press.

${ }^{2}$ Le terme "Parenthood" est celui qui a été traduit par l'anglicisme "parentalité"
} 
l'apprentissage du contrôle des pulsions. Non seulement en tant que domaine d'intérêt spécifique du psychologue, mais, aussi en tant que modalité d'apprentissage de la langue et des bases culturelles de la coutume, des gestes, et des idiomes, cette période est vitale pour la formation de la personnalité des adultes dans une société donnée. $\mathrm{Si}$, toutefois, nous sommes préoccupés par les compétences techniques et le rôle de la structure de cette même société, nous devons donner davantage de poids au contexte de l'apprentissage dans l'enfance et l'adolescence (p. 6, traduction de l'auteur) ».

La place du contexte institutionnel et social est d'autant plus importante qu'Esther Goody a pour ambition de poser un cadre général pour l'analyse et la comparaison entre différentes cultures, dont la société anglaise de la fin du $\mathrm{XX}^{\mathrm{e}}$ siècle, de décrire l'ensemble des tâches éducatives relevant non seulement de la petite enfance, mais également de l'adolescence et de considérer lesquelles reviennent aux parents selon les contextes. Pour donner toute sa place aux différents enjeux éducatifs et construire un modèle d'analyse, Esther Goody pose deux questions : «La difficulté est de définir un cadre théorique approprié. Je propose de résoudre ce problème en posant simplement la question: "Quelles sont les tâches essentielles qui doivent être menées à bien pour produire un nouveau membre de la société, pour l'élever et faire en sorte qu'il puisse y assumer les rôles d'un adulte? Que doivent faire les parents ? Il est immédiatement évident qu'il y a beaucoup de tâches qu'ils doivent accomplir et qui, dans notre société, ne sont pas menées par les parents, mais par les nourrices, les écoles, l'industrie, l'État, ou même par les nouveaux membres. » (p. 6, traduction de l'auteur)

La parentalité est donc bien envisagée de manière globale comme faisant partie des préoccupations générales d'une société à l'égard de ses membres, en vue d'assurer une partie de l'éducation des enfants de la naissance à l'âge adulte. D'emblée, la parentalité est conçue comme étant articulée aux autres institutions sociales, ce que l'on peut retrouver ultérieurement en sciences de l'éducation dans la notion de " $\operatorname{coéducation}^{3} »$. Ce poids du contexte social me semble également très fécond pour rendre compte des variations observables selon les milieux sociaux, au sein de notre propre société.

Selon Esther Goody, la parentalité recouvre différentes tâches au nombre de cinq: Procréer et engendrer ; Nommer et établir le statut de l'enfant dans la société civile et la parenté ; Remplir un ensemble de tâches quotidiennes (élever, nourrir et protéger); Assurer une

${ }^{3}$ Voir Dubet F. (1997) École, famille, le malentendu. Paris : Éditions Textuels.

Durning P. (1999). Le partage de l'action éducative entre parents et professionnels. Relevé des travaux en langue française sur la coéducation des enfants. Vaucresson, CNFE-PJJ. 
formation ; Être tuteur de l'enfant jusqu'à l'âge adulte (droits, devoirs, responsabilités). Dans la suite du propos d'Esther Goody donne des précisions sur chaque tâche, mais surtout envisage la réciprocité entre tâches parentales et devoirs filiaux. Après l'importance donnée au contexte social, il me semble qu'il s'agit là d'une deuxième spécificité de l'approche anthropologique d'Esther Goody très utile à l'exploration de la parenté en milieu populaire. En effet, comme l'illustrent les textes de Claudia Fonséca ${ }^{4}$, quelles que soient les relations de filiation, l'éducation reçue et les tâches éducatives accomplies engagent la famille biologique et le pourvoyeur de soins dans une relation durable qui engendre des contre-dons. Cette réciprocité existe tant entre les deux familles qu'entre l'enfant et ces deux familles.

Cette spécificité contraste avec le regard psychologique sur la parentalité qui accorde beaucoup d'importance aux compétences parentales et aux répercussions de ces compétences sur la construction de l'enfant et son aptitude ultérieure à devenir à son tour un parent satisfaisant, c'est-à-dire à la génération suivante. Ici, l'anthropologie sociale ne regarde pas seulement ces retombées sur l'enfant, mais elle inscrit l'éducation de l'enfant dans un ensemble de relations au sein desquelles l'éducation de l'enfant crée des liens entre les membres de la communauté, et où il revient à l'enfant d'assumer différentes tâches en retour de l'éducation reçue. L'enfant s'acquitte de ses devoirs tout au long de sa vie en rendant lui-même une partie des services reçus pendant son éducation aux adultes qui l'ont élevé et ceci au fur et à mesure du développement de ses capacités. Cette compensation du travail éducatif des adultes va jusqu'au décès de ces personnes ayant rempli des rôles parentaux et même au-delà quand il lui revient de remplir des devoirs rituels vis-à-vis de parents défunts. Cette approche de la parentalité permet d'articuler la question des âges de la vie au sein d'une société, question renouvelée en Occident par le vieillissement de la population et les devoirs des enfants - fussent-ils adultes - vis-à-vis de parents âgés dépendants. La notion de réciprocité est également utile pour rendre compte des nombreux services rendus entre membres de la famille élargie en milieu populaire, ainsi que des services attendus des enfants à l'égard des parents durant l'enfance.

Du point de vue de l'éducation de l'enfant, l'approche ethnographique d'Esther Goody invite à considérer, non seulement comme le réceptacle d'une éducation reçue, mais comme acteur de l'éducation de ses frères et sœurs, contribuant, à sa mesure, aux charges de la famille.

\footnotetext{
${ }^{4}$ Fonséca C. (1985). Valeur marchande, amour maternel et survie : aspects de la circulation des enfants dans un bidonville brésilien. Annales, économies, sociétés, civilisations, 40, 5 p. 991-1022.

Fonséca, C. (2000). La circulation des enfants pauvres au Brésil. Une pratique locale dans un monde globalisé. Anthropologie et sociétés. Dossier Nouvelles parentés en occident, 24, 3, p. 53-73.
} 
Cette manière d'envisager l'éducation peut être en contradiction avec les nouvelles normes d'éducation qui mettent en avant l'épanouissement personnel de l'enfant : «l'enfant a le droit d'être lui-même, il doit parvenir à son plein équilibre et à l'épanouissement de sa personnalité »" Comme le fait remarquer François de Singly, ce modèle apporte son lot de tensions entre épanouissement et réussite scolaire, entre attention des parents à l'égard de chacun de leurs enfants. Dans le contexte des familles populaires, il peut également soulever des conflits entre avènement de la personnalité de l'enfant et participation de celui-ci à la survie du groupe familial.

Quelles sont les particularités de l'éducation de l'enfant en milieux populaires observées dans le quotidien? Dans le célèbre livre de Didier Houzel sur la parentalité, la place des soins corporels donnés par les parents à l'enfant n'est pas centrale. La pratique de la parentalité y est relativement peu développée et toujours reliée à la dimension psychique. «On entend ici par soins non seulement les soins physiques, qui ont bien sûr toute leur importance, mais également les soins psychiques $»^{6}$. De même, les désormais classiques douze besoins énoncés par JeanPierre Pourtois et Hugette Desmet ${ }^{7}$ laissent à l'écart les besoins physiques de l'enfant, évoquant cette question en fin de chapitre. Or, la démarche anthropologique suggère d'inverser l'approche : partir du quotidien et de la pratique pour montrer comment le symbolique s'articule avec la gestuelle des soins. N'est-ce pas en répondant aux besoins physiques de l'enfant que les parents lui apprennent les principales règles sociales explicites ou implicites?

Quels sont les résultats de mes recherches ethnographiques ${ }^{8}$ qui illustrent l'éducation quotidienne dans les familles populaires? Ces recherches ont le plus souvent porté sur des familles faisant l'objet d'interventions sociales à domicile. Ayant connaissance des travaux portant sur les modèles éducatifs selon les catégories sociales ${ }^{9}$, nous partirons d'un autre point de

${ }^{5}$ Singly (de) F. (1996). Le soi, le couple et la famille. Paris, Nathan, p. 109.

${ }^{6}$ Houzel, D. (1999). Les enjeux de la parentalité. Paris, Erès, p. 151

${ }^{7}$ Pourtois J.P., Desmet, H. (2007). L'éducation post-moderne. Paris, PUF.

${ }^{8}$ Tillard B. (2007b). Marginalité ou stigmatisation? Étude ethnographique sur la naissance en milieu urbain, Tsantsa. Revue de la Société Suisse d'Ethnologie, 12, p. 41-52.

Tillard B., Lemoine M., Bruggeman D. (2009). Itinéraire de place en place : l'exemple de Kelly. Sociétés et jeunesses en difficulté, $\mathrm{n}^{\circ} 8$. Automne 2009.

Tillard B. (2010). Échanges entre familles et professionnelles. Don et contre-dons. Ethnologie française, XI, 1, p. 131-139.

Tillard B. (2011). Frères et sœurs : une relation de parenté au cœur des enjeux de nomination de l'enfant à la naissance. Revista Italiana di Educazione Familiare, 1, p. 83-98.

Tillard B., Rurka A. (2013). Trajectoires résidentielles familiales et interventions sociales à domicile. Recherches Familiales, 10, p. 75-89.

${ }^{9}$ Gayet D. (2000). Les pratiques éducatives des familles. Paris, PUF. 
vue, celui de l'ethnologue observant les relations entre les familles et les professionnels qui interviennent chez eux, le plus souvent dans le cadre de la protection de l'enfance. C'est pourquoi cet article, s'il prend comme point de départ des descriptions approfondies de scènes du quotidien nécessite d'être considéré comme un regard sur le quotidien en milieux populaires, mais ne prétend pas résumer l'ensemble des situations possibles. Nous développerons plus particulièrement le moment de la toilette et celui du repas qui l'un et l'autre renvoient à l'usage de l'espace domestique.

\section{Hygiène corporelle et stigmatisation}

\section{Les conditions de logement}

Avant de détailler quelques éléments concernant l'hygiène corporelle, un élément contextuel s'impose pour comprendre le décalage entre les normes d'hygiène dans les familles de classe moyenne et celles des milieux populaires. En effet, dans de nombreuses situations observées, la toilette des enfants ne se réalise pas dans une maison pavillonnaire équipée d'une salle de bains. Certes, lorsque le professionnel intervient dans un contexte de classe moyenne, l'architecture de la maison permet un usage différentié des espaces : les enfants font leur toilette dans la salle de bain. Dans les situations de logement précaire, non seulement l'eau chaude n'est pas toujours disponible au robinet, mais de plus, elle se pratique dans la cuisine sans différencier les lieux destinés aux repas et ceux utilisés pour l'hygiène corporelle. Dès lors, il n'est pas rare que la toilette de l'enfant se pratique dans la cuisine, dans une bassine posée sur l'évier entre la gazinière et le frigidaire.

\section{La toilette}

L'observation ethnographique permet d'illustrer les difficultés quotidiennes auxquelles les parents de familles populaires sont confrontés dans la réalisation de leurs tâches éducatives à domicile. Prenons un exemple.

Le Pape M.-C. (2009). Être parent dans les milieux populaires : entre valeurs familiales traditionnelles et nouvelles normes éducatives. Informations sociales, $\mathrm{n}^{\circ} 154$, p. $88-95$.

Le Pape M.-C., van Zanten A. (2009). Les pratiques éducatives des familles. In Duru-Bellat M., van Zanten A. (dir.) Sociologie du système éducatif. Les inégalités scolaires. Paris, PUF, p. 185-205.

Kellerhals J., Montandon C. avec la collaboration de P.-E. Gaberel, H. McCluskey, F. Osiek et M. Sardi. (1991). Les stratégies éducatives des familles. Milieu social, dynamique familiale et éducation des pré-adolescents. Lausanne, Delachaux et Niestlé.

Pourtois J.-P. (1979). Comment les mères enseignent à leurs enfants ? Paris, PUF. 
C'est à la demande du père que les services sociaux sont intervenus dans la famille de JeanPierre et Nadia. Celui-ci voyait sa femme débordée, ne parvenant pas à s'occuper de leurs deux filles. Certes, derrière cette préoccupation du mari nous pouvons retrouver le partage sexué des tâches tel que le dépeint Olivier Schwartz ${ }^{10}$ lorsqu'il aborde la place des femmes et des mères dans la vie domestique. Jean-Pierre met la main à la pâte, mais cela ne suffit pas. Tous deux ont un passé de placement institutionnel et connaissent bien les services sociaux. Cette demande du père anticipe probablement le soutien qui aurait pu leur être octroyé en raison d'une intervention extérieure, comme le montre l'épisode correspondant à la phase d'observation participante.

La directrice d'école maternelle agacée par les remarques des autres parents fait un signalement à l'Aide sociale à l'enfance, à la veille des vacances de Noël car Marion a toujours des poux ! Il est vrai que chez Marion, depuis la rentrée des classes, l'eau chaude coule très peu, le chauffe-eau est entartré. Alors qu'à deux reprises le gardien de l'immeuble a été informé, la société responsable de l'entretien du logement ne se déplace pas. Alors on s'est habitué progressivement à cette situation qui ne permet pas à l'enfant d'être lavée et soignée de ses poux dans de bonnes conditions. Aux vacances de Toussaint, la fillette qui était presque sans parasite est allée passer plusieurs jours chez ses grandsparents. Les cousins et cousines lui ont remis quelques locataires sur la tête (explication de la famille et de la TISF). De nouveau le traitement est entrepris par la maman avec le soutien de la travailleuse familiale. En décembre, le chauffe-eau tombe définitivement en panne; les toilettes des deux enfants de quinze mois et trois ans se compliquent: en attendant qu'il soit remplacé, il faut chauffer l'eau dans les casseroles et laver les fillettes dans l'évier de la cuisine. C'est à ce moment que survient le signalement.

Laver les enfants, quoi de plus ordinaire! Cependant quand les parasites s'en mêlent, que tous les membres de la famille sont touchés et que les conditions matérielles ne sont pas réunies, l'hygiène corporelle devient un objectif difficilement atteignable. L'eau chauffée dans les casseroles et la toilette pratiquée dans l'évier mettent aussi en évidence un usage de l'espace rappelant celui qui était ordinaire trois générations plus tôt. Il renvoie à la réalité quotidienne d'un espace restreint où se tiennent différentes activités.

Certes, objectivement, les poux n'empêchent pas un enfant de grandir. Cependant, ils deviennent vite un objet de stigmatisation qui conduit lors de notre visite pour l'inscription à la halte-garderie, au refus poli de la directrice qui après une rapide évaluation de la situation (langage de la mère, aspect extérieur des enfants et de la mère) refuse d'intégrer les enfants, une demi-journée chaque semaine, au prétexte que la période des inscriptions est terminée. L'intervention de l'assistante sociale de circonscription permettra de dépasser cet obstacle. Cependant, plus tard, la présence des poux est le motif du signalement effectué par la directrice

\footnotetext{
${ }^{10}$ Schwartz O. (1990). Le monde privé des ouvriers. Hommes et femmes du Nord. Paris, PUF.
} 
de l'école maternelle sous la pression des parents d'élèves. À la halte-garderie, comme à l'école maternelle, les professionnelles responsables de la structure sont dans une position de garant de la qualité de vie au sein de la structure, voire porte-parole des professionnels et des usagers de la structure qui ne souhaitent pas risquer d'être contaminés.

L'intervention sociale mise en place à la demande du père ne parvient pas à prendre en considération l'ensemble des difficultés familiales. Le travail de la TISF est pensé en première intention comme une intervention auprès de l'enfant et pour l'enfant. Secondairement il est aussi considéré comme intervention auprès des parents dont le bénéfice rejaillira sur l'enfant. Cependant il se heurte au fait qu'il prend en considération les besoins de l'enfant, sans toutefois prendre en considération les préalables à la satisfaction de ces besoins. Ainsi, l'intervention de la TISF ne considère pas comme prioritaire de régler les problèmes matériels liés au logement. Cette situation rencontrée à plusieurs reprises illustre que la segmentation des activités entre les intervenants sociaux a pour conséquence de ne pas tenir compte de certains éléments pourtant déterminants.

Les enfants portent des poux durant toute la durée de ma présence ( 3 mois), mais bien davantage selon les propos de la TISF, elle-même concernée par les parasites depuis le début de l'intervention, avant que je ne sois à mon tour touchée. La durée et l'intensité de la parasitose suggèrent que l'éducation de ces deux jeunes enfants comprend un apprentissage particulier. Porter des poux dès l'âge de quelques mois et ce, durant plusieurs années, conduit à supporter des démangeaisons en permanence, des plaies du cuir chevelu, l'odeur et les effets des produits agressifs utilisés pour le traitement. Que produisent ces réalités sur la manière dont l'enfant perçoit son corps ? Quel est leur impact sur son avenir ? Quels effets ces insecticides ont-ils sur sa santé future?

Cet exemple illustre également que le contrôle social passe par des questions souvent anciennes comme celle de l'hygiène corporelle. L'écart à la norme en matière de présentation de soi se manifeste par des signes extérieurs décryptés par les professionnels. En retour, il fait probablement percevoir à l'enfant en bas âge la différence de comportement des adultes à son égard, lui signifiant le début d'une «carrière " ${ }^{11}$ d'enfant pauvre. D'autre part, ces signes stigmatisent ${ }^{12}$ les parents en associant poux, négligence et défaut dans l'exercice de la parentalité.

\footnotetext{
${ }^{11}$ Becker H. (1963), [1986]. Outsiders. Étude de sociologie de la déviance. Paris, Métaillé.

${ }^{12}$ Goffman E. (1975). Stigmate. Les usages sociaux des handicaps. Paris, Les éditions de minuit.
} 


\section{La présentation de soi}

L'étude menée auprès des élèves décrocheurs ${ }^{13}$ a permis de rencontrer une jeune fille, Kelly, dont l'hygiène corporelle et vestimentaire préoccupait l'éducatrice. Son histoire a fait l'objet d'une étude de $\operatorname{cas}^{14}$. Il est possible de mettre en parallèle les paroles de l'éducatrice à propos des enfants lors de leur premier placement, (Kelly étant âgée d'un an), avec ceux dans lesquels cette même interlocutrice expose la stigmatisation dont la jeune fille est l'objet à son insu alors qu'ensemble elles sont à la recherche d'un stage en cuisine.

"Les enfants sont passés au travers des mailles du filet de la PMI, la maman avait conçu sa fille aînée avec un cousin, et en fait cette enfant était débile, une grosse débilité. Oui, une grosse débilité. C'était une enfant qui... pissait tout droit, qui chiait tout droit, qui mangeait avec... [...]. Les enfants étaient dans un état épouvantable, on les a retrouvés avec une... des couches qu'ils avaient depuis trois jours, heu... Le garçon avait une brûlure qui n'était pas soignée, qui était toute infectée, enfin on les a emmenés dans un état épouvantable. J'ai eu honte parce que j'étais avec une stagiaire, c'était son dernier jour de stage, juste avant les vacances de Noël, et elle a pleuré, elle a pleuré, tellement c'était vraiment épouvantable quoi, l'état dans lequel on a emmené les enfants. " (L'éducatrice d'AEMO à propos du premier placement).

Le départ de la maman de 23 ans (alors mère de 4 enfants) et l'incapacité de la grand-mère de subvenir à l'éducation des 4 enfants sont à l'origine du premier placement. Cependant l'exposé des circonstances du placement fait une large place à la description des miasmes. Les règles de propreté sont largement transgressées, la propreté n'est pas apprise à ces enfants. S'ajoute à cela deux faits remarquables d'ordre sexuel : l'acte sexuel d'une jeune femme avec son cousin du même âge et l'absence de père par excès de pères (3 géniteurs différents pour ces 4 enfants). Enfin notons l'aveuglement des services de Protection Maternelle et Infantile (PMI)... tout dans cette entretien évoque l'absence de la civilisation dans cet espace sous l'emprise d'une sauvagerie incarnée par la débauche et la saleté !

Quinze ans plus tard, la même éducatrice accompagne Kelly dans sa recherche de stage.

"Alors là aussi j'ai fait le tour des restaurants la semaine dernière avec elle: la présentation, même si elle était bien habillée, elle avait une espèce de blouson avec de la fourrure, vous savez? Les poils un peu séchés sur le bas des manches... Donc moi j'ai bien vu le regard qui était porté tout le temps »; et à un moment donné j'essaye de lui redire dans la voiture: "on en a fait plus de dix, hein! Des réponses négatives », et j'essaye de lui redire: "Tu sais la présentation c'est important, hein!" Kelly quand on... on va quelque part. C'est là qu'elle m'explique l'histoire du sourire et tout ça, je lui dis : "Oui j'ai vu, tu

13 Guigue M. (dir.) (2013) Les déchirements des institutions éducatives. Jeux d'acteurs face au décrochage scolaire.Paris, L'Harmattan.

${ }^{14}$ Tillard B., Lemoine M., Bruggeman D. (2009). Itinéraire de place en place : l'exemple de Kelly. Sociétés et jeunesses en difficulté, ${ }^{\circ}{ }^{\circ}$. Automne 2009, URL : http://sejed.revues.org/index6431.html 
fais des beaux sourires. ", et puis elle me dit : "Ben je suis bien, hein? Je suis belle, hein? Aujourd'hui. " J'ai pas osé lui dire heu... "Ben non, c'est pas très clean quand même, quoi!». Je... je me suis pas autorisée à... parce que pour elle, elle était... elle était bien mise. Mais non, il était douteux le blouson quoi. " (L'éducatrice d'AEMO à propos de la recherche de stage).

L'hygiène corporelle et la présentation de soi sont ici au cœur de l'activité éducative de la professionnelle qui constate le décalage entre les normes de propreté et d'esthétique de la jeune fille et celles attendues dans l'espace professionnel. Ce jour-là, elle hésite et choisit de d'encourager la jeune fille dans ses efforts, sans toutefois lui donner les clés pour remarquer et comprendre le regard stigmatisant posé sur elle. Cette absence de prise de position explicite de l'éducatrice ne peut être comprise que si on tient compte du chemin éducatif parcouru depuis la première citation et qui se traduit dans l'attention que Kelly porte à sa toilette. Cependant, ici, les écarts entre les normes de propreté de la famille et de la société conduisent à une hygiène défectueuse et une présentation de soi qui deviennent très clairement un obstacle à l'intégration sociale de la jeune fille dans le milieu du travail.

\section{Le repas}

\section{Repas et mélange des scènes}

Après les questions d'hygiène corporelle, envisageons une autre scène de la vie quotidienne, celle du repas. D'autres écarts à la norme y sont observables.

Ainsi, à l'heure de notre arrivée, la maman Jeanne retire la couche de Michaël et le place devant son petit-déjeuner : Michaël mange les gâteaux qu'il trempe dans un bol de lait, il est assis sur le pot, lui-même posé sur une chaise placée devant la table du séjour. Jeanne nous offre un café autour de cette même table. À ce moment de la matinée, la Technicienne d'Intervention Sociale et Familiale (TISF) et elle se rappellent les rendez-vous à venir (avocat, tribunal, hôpital, Caisse d'allocation familiale, rencontre avec l'éducateur de la Maison d'Enfants à caractère social, etc.). Autour du café, elles planifient également le travail de la matinée. Les odeurs qui manifestent la satisfaction d'un besoin de l'enfant se mélangent à celle de notre café... Enfin, avant de faire la toilette et d'habiller l'enfant, Jeanne retire le pot, nettoie sommairement les fesses de l'enfant tandis que celui-ci est debout sur la chaise, puis elle vide le pot aux WC... 
Lors d'un déplacement où nous accompagnons le même enfant à la Consultation médico-psychologique où l'enfant a rendez-vous avec la psychomotricienne,

je fais remarquer (à Myriam, la TISF) que certains éléments sont inconfortables pour leur travail dans les familles (le chauffe-eau entartré, les chiens, l'état de l'armoire à pharmacie, etc.), qu'une visite «technique» pourrait favoriser la prise en charge de ces éléments par les familles. Myriam considère cela comme des sujets auxquels je suis sensible parce que sans grande expérience du métier et sur lesquels on se bat un temps, puis on capitule... à ce moment, elle prend en exemple le pot de Michaël posé sur la chaise pendant le petit-déjeuner. Elle trouve ça dangereux (risque de chute) et désagréable (odeurs), mais elle ne parvient pas à avoir l'assentiment de la maman sur un changement des habitudes. Le jour où elle voulait en parler, lors d'une synthèse, la psychomotricienne n'était pas là et elle n'a donc pas eu son soutien. Elle objecte aussi que la lettre aux HLM (qu'elle a rédigée la semaine dernière) pour le tapage nocturne se justifie en raison de l'insécurité ressentie par "Madame » et des risques de dérapage des relations entre son environnement social et elle. En revanche, pour le chauffe-eau, il faut s'adresser au concierge. « Madame » l'a déjà fait, Myriam estime que c'est de sa responsabilité, mais si les services techniques ne sont pas venus et que Jeanne - la mère - ne les relance pas, Myriam le rappellera peut-être, mais elle n'insistera pas là-dessus. ${ }^{15}$

Manger sur le pot à l'âge de cinq ans peut être considéré au même titre que faire la toilette dans l'évier. Les nouvelles normes d'habitat ont contribué à séparer la salle à manger des toilettes, ou encore dissocier la cuisine de la salle de bains. Si la TISF met en avant le risque de chute, elle n'évoque le mélange des odeurs qu'en deuxième instance, probablement moins indisposée que moi. Je suis impliquée dans cette scène, car je dois faire honneur au café qui m'est servi. Ce choc des cultures, celle de l'ethnologue et celle de la pratique d'une famille populaire dans lequel interviennent de nombreux travailleurs sociaux souligne le côté inhabituel de la situation et attire mon attention sur la particularité de l'apprentissage de l'enfant qui est en train de se jouer. Comme dans la question des poux, cette particularité éducative n'a pas de retentissement immédiat sur la santé ou le développement de l'enfant, mais elle concerne ses apprentissages. Je souligne donc que l'expérience corporelle de l'acte de manger est associée, chaque matin où il m'est permis de l'observer, à l'apprentissage de la propreté et donc à la satisfaction simultanée des besoins d'uriner ou de déféquer, et ce, en public, c'est-à-dire devant la mère, la TISF et éventuellement la sœur, la personne de passage dans l'appartement.

Cet extrait conduit à prendre en considération la construction sociale progressive de l'intolérance à certaines odeurs telle qu'elle est décrite par Alain Corbin dans Le Miasme et la

\footnotetext{
15 Tillard B. (2004) Observation ethnographique des interactions TISF-Familles, Rapport final PRS 2000336 Santé des jeunes, Uriopss Nord Pas-de-Calais, 107p. (possibilité de consultation sur la site http://www.oned.gouv.fr/etudes-recherches-liste-recherches.html).
} 
jonquille $^{16}$. De plus, c'est la deuxième évocation de l'utilisation de l'espace privée où les activités se mélangent ne peut être passée sous silence, tant ces normes sociales sont hautement investies par les acteurs dans différents registres comme le souligne Marie Douglas, dans son essai De la souillure ${ }^{17}$.

Le contexte familial doit être précisé pour mieux comprendre la tolérance de la TISF. De multiples objectifs sont menés de front par la TISF dans son intervention auprès de cette famille monoparentale de huit enfants. Les deux aînés ont quitté le domicile maternel. Cinq enfants entre 7 et 17 ans sont placés en maison d'enfants à caractère social (MECS). Seul le dernier reste quotidiennement auprès de sa mère, mais Myriam participe aussi à l'accueil des enfants lors des week-ends et des vacances quand les enfants placés viennent chez leur mère. Elle est donc souvent contrainte à effectuer des choix. Ainsi, en dehors des périodes d'accueil des plus grands, elle privilégie le soutien de Jeanne dans les démarches de demande d'aide juridictionnelle pour sa fille qui a subi des violences sexuelles, dans les démarches pour la constitution d'un dossier de demande d'allocation d'éducation spéciales (AES) et demande d'intervention du Service d'Éducation Spéciale et de Soins à Domicile (SESSAD) ainsi que le suivi médico-psychologique de Mickaël qui est une mesure transitoire en attendant le SESSAD.

Par ailleurs, durant ces trois mois d'observation à raison d'une demi-journée par semaine, je n'ai rencontré aucun des nombreux travailleurs sociaux concernés par cette famille. À travers ces précisions, je ne cherche pas à justifier la professionnelle, mais d'une part à émettre l'hypothèse d'une substitution des tâches des travailleurs sociaux à la TISF et d'autre part à donner quelques éléments du contexte institutionnel qui situe le face à face dans un ensemble de contraintes qui influencent la nature de l'aide éducative apportée à la mère. Le souci d'anticipation est ici centré sur l'accomplissement de tâches institutionnelles dont les échéances sont fixées (établir un dossier de demande d'aide juridictionnelle pour qu'un avocat puisse accompagner un enfant de la fratrie abusé par son instituteur) et/ou inscrites dans le contrat entre le Conseil général et l'association employant la TISF (obtenir une intervention du SESSAD et une AES). En revanche les tâches éducatives centrées sur les apprentissages concrets de l'enfant n'apparaissent pas au centre de l'activité, à l'exception des remarques de la TISF concernant le langage de la mère s'adressant à son enfant.

${ }^{16}$ Corbin A. (1982). Le Miasme et la jonquille. Paris, Flammarion.

${ }^{17}$ Douglas M. (2001) (1ère éd. 1966). De la souillure. Essai sur les notions de pollution et de tabou. Paris, La Découverte. 


\section{Le repas des enfants et le rôle des aîné(e)s}

Dans la gestion du quotidien, chacun prend rapidement une part afin d'assurer un fonctionnement familial satisfaisant pour chacun. Ainsi, un matin lorsque nous arrivons chez Christine, trois fillettes sont attablées autour du petit déjeuner que la plus âgée a servi en attendant l'aide de la TISF. La plus jeune est assise dans sa chaise, elle trempe son pain et mange dans sa chaise haute, un peu en retrait de la table. La mère qui travaille comme animatrice de promotion en supermarché à quarante kilomètres de là est partie tôt. L'aîné, un garçon plus âgé est déjà au collège. La famille monoparentale occupe un préfabriqué datant de l'après-guerre et situé au fond du jardin des grands-parents maternels. C'est un deux pièces (plus petite cuisine avec douche. Les toilettes sont séparées et placées à l'autre extrémité du logement). Le logement est chauffé par un petit poêle à bois et charbon dans le séjour. Six personnes et un berger allemand y vivent.

Ici pour pouvoir travailler, la mère doit compter quotidiennement sur l'aide des aînés. La responsabilité des plus grands, ici plus particulièrement de l'aînée des filles, s'impose comme une valeur familiale transmise en milieu populaire, même si par ailleurs l'égalité de traitement est affirmée. $^{18}$

\section{Le repas des enfants : disette, partage et solidarités}

Si l'observation permet de rendre compte de l'organisation du repas, elle permet également de nous intéresser à son contenu. Voici un repas d'enfants observé dans une autre famille, chez Louis et Marie.

Dans cette cuisine d'HLM, les enfants se disputent pour un problème de nombre de places assises autour de la table. Il y a un banc et la place pour 3 chaises en les serrant bien. Le thème de dispute est fréquent au moment du repas : c'est un petit espace repas idéal pour 4 mais ils sont toujours davantage à manger ensemble : les 5 enfants de la famille mangent en même temps. Ils sont souvent accompagnés du fils d'une amie qui a déménagé et habite maintenant loin de l'école. Ils préfèrent les places sur les chaises plutôt que celles sur le banc où il faut se serrer pour que chacun puisse être assis.

Une autre dispute survient à propos d'une assiette « Mickey ».

«Allez tout le monde assis ! ». Le père prend la distribution du repas en charge.

Malheureusement, le plat de pâtes cuisinées et surgelées n'est pas assez chaud, le père réchauffe les assiettes une à une au four à micro-ondes. Pendant ce temps,

\footnotetext{
${ }^{18}$ Tillard B. (2002). Ce qu'il en coûte de nourrir. In D. Bonnet, C. Le Grand - Sébille, M.F. Morel (dir.) Allaitements en marge. Paris, L'Harmattan, p. 23-47.
} 
l'impatience des enfants s'accroît. Ceux qui attendent regardent manger celui dont l'assiette est chaude...

Pour le dessert, quatre clémentines sont partagées entre les enfants, d'autres se contentent d'un biscuit au chocolat.

Pour le repas, les enfants ont donc mangé à eux six, un kilo de pâtes cuisinées surgelées. Quant aux parents, ils n'ont pas pris de repas. La mère a mangé une tartine avec du fromage, le père n'a rien pris alors qu'il a travaillé tôt ce matin. Il n'y a plus rien dans le frigidaire. Il reste un peu de pain (le fait que la mère a refusé de donner cinq tartines à une voisine revient à deux reprises dans la conversation). ${ }^{19}$

Durant la majeure partie de la scène, la TISF parle avec la mère (environ 35 ans). Ce matin, le médecin est passé pour l'examiner. Elle tousse et crache du sang depuis plusieurs jours. Elle doit se rendre en consultation au cabinet médical pour un test de dépistage de la tuberculose et à l'hôpital en consultation ORL. La mère, fumeuse, redoute l'endoscopie du larynx et des bronches, mais également le sermon du médecin spécialiste qui lui a recommandé à plusieurs reprises l'arrêt du tabac. La TISF tente de dédramatiser l'examen en faisant référence à son expérience personnelle et en l'engageant à prendre rendez-vous.

Chez Louis et Marie comme chez Nadia et Jean-Pierre, le père s'implique dans les activités domestiques. Ici il fait face à l'accablement de sa femme et à l'énervement de ses quatre garçons et de sa fille, il se charge du repas. Nous sommes à quelques jours de Noël et le frigidaire est quasiment vide. Qui donc a eu l'idée de placer Noël en fin de mois ? Les rations servies sont insuffisantes. Les parents qui sont engagés dans le remboursement de dettes de la grand-mère maternelle ne sont pas prioritaires. Les voisins non plus! Aujourd'hui on ne partagera pas le reste de pain. La famille utilise pourtant différentes stratégies. Le père est volontaire au secours populaire et obtient ainsi quelques denrées alimentaires. À certains jours, la mère envoie chaque enfant à un étage différent de l'immeuble pour demander l'un des œufs, un autre de la farine, un autre du lait, un quatrième du sucre et prépare ainsi des crêpes pour toute la famille. En retour, certains voisins la sollicitent, mais aujourd'hui impossible de répondre positivement.

La solidarité a des limites ! Cependant, elle continue en partie de s'exercer à l'égard du fils d'une ancienne voisine, copain des enfants qui s'invite très souvent chez eux. Le partage s'exerce tant en ce qui concerne l'espace que les vivres. N'idéalisons pas la scène ! Les enfants crient, se disputent pour les meilleures places, les parts d'apparence un moins maigres, tandis que la mère proteste en aparté sur l'absence de participation de l'ancienne voisine aux frais de repas

${ }^{19}$ Tillard B. (2004) Observation ethnographique des interactions TISF-Familles, Rapport final PRS 2000336 Santé des jeunes, Uriopss Nord Pas-de-Calais, 107p. (possibilité de consultation sur la site http://www.oned.gouv.fr/etudes-recherches-liste-recherches.html), p. 37-39. 
de son fils. Néanmoins, malgré la disette, les quelques 1500 Calories ont été partagées le plus équitablement possible entre les six enfants.

Par ailleurs, je n'ai assisté à aucun repas pris en commun et réunissant parents et enfants. Même lorsque les enfants sont assez grands pour manger sans l'aide d'un adulte, le plus souvent les parents mangent après les enfants. Dans le cas présent, la séparation du repas des enfants et des adultes semble rendre moins perceptible aux enfants l'insuffisance d'espace et l'insuffisance de nourriture, mais ce type d'organisation souligne aussi l'écart entre les deux générations qui cohabitent quotidiennement. 


\section{Frères et sœurs partagent l'espace}

Une autre caractéristique de l'utilisation de l'espace dans la vie quotidienne est que les frères et sœurs partagent généralement leur chambre. Parfois, les parents organisent une chambre de filles et une chambre de garçons (ex. Louis, Marie et leurs 5 enfants en F4 d'HLM : chambre de la fille, chambres de garçons, chambre des parents), mais parfois l'espace ne permet pas cet aménagement (Christelle et ses 5 enfants dans le deux pièces : chambre des enfants, mère dans un débarras étroit et froid, près des toilettes ou dans le séjour selon la température). Les filles dorment alors sur des lits superposés, le garçon sur le côté dans un lit isolé. Dans la famille de Kelly, la mère dort avec les filles et le mari avec les garçons.

Les familles populaires dont il est question ici, familles souvent nombreuses et sans travail, subissent leurs conditions de logement, s'y adaptant avec l'espoir d'y remédier, espoir souvent repoussé d'année en année. Une nouvelle fois, l'observation nous confronte à un écart entre familles populaires et familles des classes moyennes qui a priori n'est pas majeur... partager la chambre n'empêche pas de grandir. Il est possible de rapprocher ce constat de l'éducation reçue par nos parents ou grands-parents dans la première moitié du XXe siècle. Des familles plus aisées vivent dans les centres urbains où la surface au $\mathrm{m}^{2}$ ne permet pas d'appliquer la norme d'un enfant par chambre et d'une chambre pour les parents. Cependant, ces cas ne font pas l'objet d'un regard négatif quand ces familles de classes moyennes ou supérieures, moins soumises au regard des travailleurs sociaux peuvent mettre à profit les avantages d'une situation urbaine qui facilite leur accès aux activités culturelles et aux établissements scolaires renommés, ou encore, lorsqu'elles peuvent envisager d'autres modes de vie (appartement plus loin du centre, mais plus grand), etc. Ce mode d'occupation de l'espace en milieux populaires a pour conséquence un regard particulier d'autrui sur l'éducation des enfants.

\section{Le regard social sur l'espace}

La promiscuité a longtemps été un souci des hygiénistes et des personnes luttant contre la tuberculose. La pauvreté associée à la promiscuité, à l'alcoolisme, au défaut d'aération des logements et de propreté étaient des thèmes des campagnes de prévention et des visites sanitaires au domicile des familles.

De nos jours, sous le regard de travailleurs sociaux ou de professionnels de santé, les précisions concernant l'habitat sont apportées quand il s'agit de décrire ou d'argumenter des signalements. Ainsi, la sociologue Delphine Serre analysant les signalements d'enfants en 
danger, relève que l'espace domestique est évoqué quand le rédacteur du signalement souhaite exprimer par un euphémisme le désordre sexuel vécu au sein d'une famille, comme l'« étalage de leurs vies intimes devant les enfants $»^{20}$. Dans d'autres circonstances, en l'absence d'aveux, la description de la chambre, des lits et des objets découverts (p. 107) deviennent des éléments permettant de relever des indices au sein du domaine privé par lesquels le travailleur social fait part de ses soupçons ou rédige et appuie les informations transmises par des tiers. Par ailleurs, Delphine Serre souligne que les assistantes sociales véhiculent une « morale familiale de classes moyennes salariées » (p. 124) qui s'exprime dans la norme d'individualisation de l'enfant quel que soit son âge, au sein de la fratrie. Or, « la réalisation effective de cette norme repose [...] sur des conditions sociales et économiques particulières» (p. 125) et conduit indirectement «à dévaloriser les familles nombreuses » (p. 126).

De même les psychologues de crèches ou les puéricultrices peuvent insister sur « la difficulté à dégager un « espace vital» dans un «appartement excessivement encombré d'objets de toutes sortes » (p. 119-120). Cette citation évoque le mélange des objets de provenance et de fonctions variées, comme il a été souligné précédemment, ce désordre n'est pas seulement un argument matériel, mais il tend à évoquer d'autres désordres pour le lecteur (le juge, le responsable de l'ASE). La place de l'espace physique de l'enfant dans la maison ou l'appartement est cependant un argument mineur, tant la place métaphorique est souvent au premier rang des arguments évoqués. La place de l'enfant, l'intérêt des parents pour son éveil, la satisfaction de ses besoins personnels, le soutien, les encouragements, le respect dont il est l'objet, sont davantage au centre du signalement.

\section{Impact sur le travail scolaire}

Les espaces de la jeunesse des quartiers sensibles sont souvent traités en opposant l'espace domestique intérieur et l'espace public extérieur. De même, Richard Hoggart et Olivier Schwartz traitent des questions d'espace en opposant univers masculin (extérieur) et univers féminin (intérieur). Cependant, dans ces travaux, l'organisation de l'espace intérieur y est peu abordée. Olivier Schwartz traite de la place des hommes dans l'intérieur ouvrier du bassin minier du Nord-Pas-de-Calais régenté par la mère. La place du père est évoquée à propos de la question du bricolage qui peut prendre des formes utilitaires ou créatives et à propos de leur rôle vis-à-vis

\footnotetext{
${ }^{20}$ Serre D. (2009). Les coulisses de l'Etat social. Enquête sur les signalements d'enfant en danger. Paris, Raisons d'agir, p.105
} 
du travail de l'école. La scène dans laquelle le père décrète que son fils doit faire des progrès en français et où il intervient dans la rédaction de son fils sous les yeux de l'enquêteur et de la mère, évoque une scène qui se passerait sur la table de la cuisine ou du séjour, mais la topographie n'est pas précisée $e^{21}$.

Or, ces observations de l'espace domestique soulèvent la question des retombées de cet usage de l'espace domestique sur l'éducation de l'enfant et plus particulièrement sur le travail scolaire de l'enfant. Comment l'enfant peut-il apprendre des leçons ou rédiger un devoir s'il ne peut s'installer quelque part? Certes, nous connaissons des enfants de classes moyenne ou supérieure qui vont préférer apprendre dans le brouhaha du séjour, sur un coin de table devant la télévision ou rédiger sur leurs genoux assis sur le lit... mais la plupart des enfants de ces classes sociales qui utilisent ces méthodes ont la possibilité de choisir leurs modalités de travail et d'alterner avec d'autres moments d'isolement. Comme pour d'autres domaines (le recours aux médecins, les structures scolaires de proximité, l'habitat ${ }^{22}$ ), la principale caractéristique de la pauvreté, ce n'est pas nécessairement le manque, mais plutôt l'absence de choix ou, disons, la soumission à des contraintes matérielles qui rendent le choix impossible. D'une certaine manière, ce partage de la chambre prépare les enfants à supporter cette absence de choix qui se poursuivra dans les contraintes d'un habitat précaire, voire dans d'autres domaines de sa vie sociale à venir.

\section{Tension entre égalité et hiérarchie}

Par ailleurs, la norme d'individualisation de l'espace et des pratiques éducatives à l'égard de chacun des enfants de la fratrie s'oppose à l'importance dans la culture ouvrière de traiter les enfants de manière égale, comme membre de la fratrie, les frères et sœurs constituant un groupe différentié de celui des adultes. L'exemple du repas pris en deux services, celui des enfants précédant celui des adultes illustre cette distinction entre les générations.

Le traitement à l'identique des enfants est une préoccupation souvent constatée, par exemple en termes de nomination où le capital symbolique des prénoms attribués va tenir compte à la fois de l'équilibre des apports entre les conjoints, mais également de l'équité entre les attributs de chacun des membres de la fratrie. Le même souci anime les parents, et plus particulièrement les mères lorsqu'elles décident du mode d'alimentation de leur enfant

${ }^{21}$ Schwartz O. (1990). Le monde privé des ouvriers. Hommes et femmes du Nord. Paris, PUF, p. 414.

22 «Les gens sont dépossédés de leur pouvoir de décision, de leur capacité d'invention, de leur volonté. Leur sort leur échappe : «On ne sait pas où ils vont nous mettre après. » (Pétonnet, C., 1985, On est tous dans le brouillard. Ethnologie des banlieues. Paris, Galilée, p. 62) 
(allaitement maternel ou biberon; moment de la diversification de l'alimentation), se référant toujours à ce qui a été fait pour les aînés lorsqu'elles exposent leurs choix pour les derniers de la fratrie. Cependant, lorsque les mères s'appuient sur les aînés pour gérer le quotidien, il n'est pas rare que des entorses soient faites à ce principe. Ainsi, plus particulièrement dans les familles monoparentales, les aînés sont souvent sollicités pour donner leur avis en matière de nomination d'un nouveau-né ou tenir la place d'un parrain ou d'une marraine. Dans un contexte de nécessité, une certaine hiérarchie entre les frères et sœurs s'établit : les aînés participent aux prérogatives des adultes. Les plus grands sont sollicités non seulement dans les aspects symboliques, mais également dans la vie quotidienne pour participer à l'entretien domestique et à la garde des plus jeunes. Sans doute l'enfance est-elle alors moins pour ces enfants «un état de protection et d'insouciance $»^{23}$ qu'un moment d'apprentissage de ce rôle de protection à l'égard des plus jeunes au sein de la fratrie. Les derniers d'une famille sont à leur tour sollicités pour participer aux soins de leurs neveux et nièces, enfants de leurs aînés. Se poursuit ainsi des échanges quotidiens qui animent le réseau de parenté ${ }^{24}$. De plus, en matière de hiérarchie fraternelle, les filles, davantage que les garçons, participent progressivement aux tâches domestiques (vaisselle, lit, cuisine) et contribuent aux solidarités familiales dans le cadre de la famille élargie, comme l'ont souligné Blandine Mortain et Cécile Vignal dans leur enquête sur la décohabitation des jeunes inscrits à la Mission Locale de Roubaix ${ }^{25}$. Ainsi, si les différences entre générations restent très marquées, il n'est pas rare que des enfants suppléent aux parents en occupant momentanément leurs fonctions telle que la présence auprès des plus jeunes, ou encore, comme cela a été mentionné précédemment, la participation aux stratégies de survie dans les moments de disette. Ainsi, à la fois pensé comme catégorie distincte de l'âge adulte, l'enfance en milieu populaire est un terrain d'apprentissage des tâches attendues des adultes par l'expérience au sein de la famille. Ces variations et ces contradictions sont une raison supplémentaire de penser les milieux populaires au pluriel.

${ }^{23}$ Delalande J. (2010) Comment des enfants et des adolescents voient-ils les âges de la vie ? Le Témémaque, 37, 1, p. 74.

${ }^{24}$ Weber F. (2013). Penser la parenté aujourd'hui. La force du quotidien. Paris, éditions rue d'Ulm.

${ }^{25}$ Mortain B., Vignal C. (2013). Processus de décohabitation en milieux populaires. Le poids des rôles familiaux de substitution sur les parcours féminins. Agora débats/jeunesses, 63, p. 23-35. 


\section{Conclusion}

Avec Séverine Gojard analysant les pratiques d'alimentation durant la prime enfance, nous constatons la diversité des modèles d'enfance dans la France contemporaine ${ }^{26}$. Les contraintes de l'espace domestique et l'organisation familiale ont été abordées à partir de l'exemple de la toilette et du repas, illustrant le rôle de l'espace domestique dans la socialisation primaire de l'enfant. Ainsi, les familles disposant de faibles ressources socio-économiques ont souvent un logement inadapté au regard des normes actuelles d'habitat. Elles organisent des activités éducatives variées dans un même lieu. Par ailleurs, l'enfant partage sa chambre et, en dehors de son lit, il ne dispose pas de lieu qui lui soit dédié. Il vit en permanence au contact de ses proches en partageant l'espace commun. Ces éléments font que l'éducation reçue par ces enfants, est alors plutôt conforme à un modèle d'enfance où l'enfant appartient à un groupe, ici le groupe familial ${ }^{27}$, nommé par Doris Bonnet, Catherine Rollet et Charles-Edouard de Suremain, modèle de l'enfant du lignage, plutôt qu'à un modèle cultivant la singularité de l'enfant vu « comme une personne ». L'enfant est éduqué, il grandit et est valorisé rarement pour ce qu'il fait d'unique, mais plus souvent pour ce qu'il apprend et réalise pour le groupe. Au sein du groupe familial, sont particulièrement valorisés les apprentissages utiles à la maisonnée ou à la famille élargie.

A contrario, l'organisation domestique des familles plus aisées et privilégiant le modèle de "l'enfant comme personne » est congruente avec le fait que les enfants sont encouragés à développer leurs talents individuels et à les affirmer au sein de la famille. Cette perspective passe par des évolutions architecturales qui se sont progressivement affirmées au cours du XXe siècle $^{28}$. La chambre est alors un des espaces marquants de cette transformation de l'enfant puis de l'adolescent, de cette socialisation primaire des relations à soi ${ }^{29}$ et des relations aux autres ${ }^{30}$. La salle de bain en est un autre où chacun constate visuellement les transformations du corps, où

${ }^{26}$ Gojard, S. (2012). L'alimentation dans la prime enfance, un révélateur de modèles de l'enfance dans la France contemporaines. In Bonnet D., Rollet, C., Suremain (de) C.-E., (2012). Modèles d'enfance. Successions, transformations, croisements. Paris, editions des archives contemporaines.

${ }^{27}$ Bonnet D., Rollet, C., Suremain (de) C.-E., (2012). Modèles d'enfance. Successions, transformations, croisements. Paris, editions des archives contemporaines, $\mathrm{p} .15$.

${ }^{28}$ Legros-Bawin B., Dandurand R., Kellerhals J. de Singly F. (dir.) (1995). Les Espaces de la famille, actes du colloque de l'AISLF, Liège, 5-6 mai 1994.

${ }^{29}$ Glevarec, H. (2010). Les trois âges de la "culture de la chambre". Ethnologie Française, XI, 1, p. 19-30.

${ }^{30}$ Poittevin A. (2005). Une lecture des espaces pour lire les liens fraternels, Espaces et societies, $2, \mathrm{n}^{\circ} 120-$ 121, p. 61-77. 
il compose avec l'image de soi, pour soi et pour autrui ${ }^{31}$ aux différents âges de la vie. Dans les situations de précarité rencontrées lors de l'entrée au domicile de familles faisant l'objet d'interventions sociales, certains préalables ne sont pas remplis pour que se déploie l'individualité de chacun. Comment s'exercer au maquillage ou pratiquer une toilette intime à l'abri des regards, avec la certitude de ne pas être dérangé, lorsque les espaces de la cuisine et de la salle de bains se confondent, lorsque la chambre sert également de salon ou est partagée par trois ou quatre enfants? Aucun paravent ne garantira les explorations d'un enfant ou d'un adolescent aussi bien qu'un verrou poussé ou un tour de clé donné en rentrant dans sa salle de bain ou la chambre. Dans le prolongement de ces constats, sans doute est-il utile de considérer les apprentissages spécifiques permis par les espaces restreints dont les familles disposent : l'enfant apprend à partager ses espaces avec ses proches et à considérer chaque espace comme remplissant plusieurs fonctions, ce qui confère une forme d'adaptation qui lui sera utile lors des trajectoires résidentielles aux déménagements fréquents pour trouver un logement peu coûteux et mieux adaptét ${ }^{32}$.

La place occupée dans mes travaux par les interventions sociales en direction des familles font également apparaître les enfants des familles populaires comme des enfants « de la Nation ». Qu'il s'agisse de veiller à ce qu'il ne soit pas maltraité, de se préoccuper de sa sécurité et d'un minimum de confort, ou encore de s'assurer de sa fréquentation scolaire, l'enfant n'est plus seulement regardé comme l'enfant d'une famille, mais comme celui d'une Nation responsable de l'application des Droits de l'Enfant et veillant à la «protection de l'enfance ».

${ }^{31}$ Mardon, A. (2009). Les premières règles des jeunes filles: pubeté et entrée dansl'adolescence, Sociétés contemporaines, 75 , p. 109-129.;

Diasio, N. (2012) Maillage des temps et gouvernement des corps dans la construction des rapports d'âge et de genre, SociologieS [En ligne], Dossiers, Genre et vieillissement, mis en ligne le 15 novembre 2012, http://sociologies.revues.org/4118

Vinel V. (2012). Discours et pratiques autour des soins du corps chez les enfants de 9 à 13 ans, communication aux Journées scientifiques Pôle Suds/Ceped, "Filles et garcons: regars croisés sur l'enfance et le genre", CEPED-INED, Paris, 5-6 juin 2012.

${ }^{32}$ Tillard B., Rurka A., (2013). Trajectoires résidentielles familiales et interventions sociales à domicile. Recherches Familiales, 10, p. 75-89. 
